\title{
A note on Euler approximations for stochastic differential equations with delay
}

\author{
Istvan Gyöngy and Sotirios Sabanis * \\ Maxwell Institute for Mathematical Sciences and School of Mathematics, \\ University of Edinburgh, Edinburgh EH9 3JZ, U.K.
}

August 7, 2018

\begin{abstract}
An existence and uniqueness theorem for a class of stochastic delay differential equations is presented, and the convergence of Euler approximations for these equations is proved under general conditions. Moreover, the rate of almost sure convergence is obtained under local Lipschitz and also under monotonicity conditions.

Keywords: Stochastic delay differential equations, Euler approximations, rate of convergence, local Lipschitz condition, monotonicity condition.
\end{abstract}

AMS subject classifications: $60 \mathrm{H} 99$

\section{Introduction}

Stochastic delay differential equations (SDDEs) play an important role in understanding and modelling many real world phenomena for which the principle of causality does not apply. One could refer to [16], 4], [17] and [1 for applications in biology, ecology, economics and finance to name a few, without, of course, exhausting the long list of the existing literature on the subject matter. It is important therefore to determine precisely under which conditions one obtains a unique solution for a delay system and, moreover, to study the convergence of suitable numerical schemes. To this end, we employ techniques from the theory of stochastic differential equations (SDEs) with random coefficients so as to determine the conditions for uniqueness and existence of solutions of delay models. Furthermore, we investigate the convergence properties of Euler schemes that are used to approximate the aforementioned models.

Strong discrete-time approximations of SDDEs (in $L^{p}$-sense) have been studied by several authors, including [13], 2], [14] and [9] amongst others. Moreover, in recent years, new findings appeared in the direction of week approximations, see for example [3].

\footnotetext{
${ }^{*}$ Corresponding author. Email: s.sabanis@ed.ac.uk
} 
Our reason for presenting here results on two types of convergence, almost sure and in probability, for numerical schemes of delay models is twofold.

First we contribute to the understanding of delay models by providing new results while imposing essentially weaker conditions on the smoothness of the coefficients in comparison with the current literature, see for example [2], [3], [13], [10] and [14] and the references therein. The convergence of Euler approximations is proved under local monotonicity condition, which is much weaker than the local Lipschitz condition that appears in [14]. Moreover, no smoothness condition on the initial data, on the delay function and on the drift and diffusion coefficients in the delay argument are assumed in order to obtain the convergence in probability. The main result of [14], Theorem 2.1, states convergence in mean square. It should be noted, that under condition $\left(H_{3}\right)$ used in [14, our convergence results clearly imply convergence in mean square as well. In addition, under local monotonicity condition we present the almost sure rate of convergence of Euler approximations whereas, Theorem 2.5 in [10] requires global Lipschitzness.

Second, we facilitate the development of the theory with regards to the understanding of quantitative and qualitative characteristics of solutions of delay equations. As an example, one may consider the study of different types of stability (almost sure asymptotic, exponential, mean square etc) for solutions of such models. This is an area which has attracted significant attention in recent years, see for example [15], [8], [19] and the references therein.

Finally, we note that although the authors in [18] provide an existence and uniqueness theorem for stochastic functional differential equations, a more general class of SDDEs than ours, their conditions are stronger in comparison with Theorem 2.1 below. The reason for this is that we do not require any smoothness of the drift and diffusion coefficients in the arguments corresponding to the delays.

We conclude this section by introducing some basic notation. Let $x y$ be the scalar product of vectors $x, y \in \mathbb{R}^{d}$ and $|x|$ be the length of $x$. Moreover, if $g \in \mathbb{R}^{d \times m}$ is a matrix, then let $g^{T}$ and $|g|$ denote the transpose of $g$ and the Hilbert-Schmidt norm respectively, i.e. $|g|=\sqrt{\operatorname{tr}\left(g g^{T}\right)}$. In addition, let $[x]$ denote the integer part of the real number $x$. Finally, let $\mathcal{P}$ and $\mathcal{B}(V)$ denote the predictable $\sigma$-algebra on $\mathbb{R}_{+} \times \Omega$ and the $\sigma$-algebra of Borel sets of topological spaces $V$ respectively.

\section{Main Results}

Let $\beta\left(t, y_{1}, \ldots, y_{k}, x\right)$ and $\alpha\left(t, y_{1}, \ldots, y_{k}, x\right)$ be $\mathcal{B}\left(\mathbb{R}_{+}\right) \otimes \mathcal{B}\left(\mathbb{R}^{d \times k}\right) \otimes \mathcal{B}\left(\mathbb{R}^{d}\right)$-measurable functions with values in $\mathbb{R}^{d}$ and $\mathbb{R}^{d \times m}$, respectively. Consider the stochastic delay differential equation

$$
\begin{aligned}
d X(t) & =\beta\left(t, X\left(\delta_{1}(t)\right), \ldots, X\left(\delta_{k}(t)\right), X(t)\right) d t+\alpha\left(t, X\left(\delta_{1}(t)\right), \ldots, X\left(\delta_{k}(t)\right), X(t)\right) d W_{t}, \\
X(t) & =\xi(t), \quad \forall t \in[-C, 0]
\end{aligned}
$$

on a fixed probability space $(\Omega, \mathbb{F}, \mathbb{P})$, equipped with a right-continuous filtration $\mathbb{F}:=$ $\left\{\mathcal{F}_{t}\right\}_{t \geq 0}$ and an $m$-dimensional Wiener martingale $W:=\left\{W_{t}\right\}_{t \geq 0}$, where $\xi:=\{\xi(t)\}_{t \in[-C, 0]}$ is a continuous process which is $\mathcal{F}_{0}$-measurable for every $t \in[-C, 0]$ for a fixed constant 
$C>0$, and $\delta_{i}(t)$ is an increasing function of $t$ such that $-C \leq \delta_{i}(t) \leq\left[\frac{t}{\tau}\right] \tau$ for some positive constant $\tau$ and $1 \leq i \leq k$. Note that two popular cases for $\delta_{i}$ are included here. These are the fixed delay case, $\delta_{i}(t)=t-\tau$, and $\delta_{i}(t)=\left[\frac{t}{\tau}\right] \tau$ which appear in many applications, see for example [1].

Fix a constant $T>0$. Let $\mathbb{L}$ denote the set of nonnegative integrable functions on $[0, T]$ and $y:=\left(y_{1}, \ldots, y_{k}\right)$. Consider the following conditions:

$\left(C_{1}\right)$ The function $\beta(t, y, x)$ is continuous in $x$ for any $t$ and $y$.

$\left(C_{2}\right)$ For every $R>0$, there exists a $K_{R} \in \mathbb{L}$ such that for all $t \in[0, T]$

$$
\sup _{|x| \leq R} \sup _{|y| \leq R}|\beta(t, y, x)| \leq K_{R}(t)
$$

$\left(C_{3}\right)$ For every $R>0$, there exists a $L_{R} \in \mathbb{L}$ such that

$$
2(x-z)(\beta(t, y, x)-\beta(t, y, z))+|\alpha(t, y, x)-\alpha(t, y, z)|^{2} \leq L_{R}(t)|x-z|^{2},
$$

for $t \in[0, T]$ and $|x|,|y|,|z|<R$

$\left(C_{4}\right)$ For any $R>0$, there exists a $M_{R} \in \mathbb{L}$ such that

$$
2 x \beta(t, y, x)+|\alpha(t, y, x)|^{2} \leq M_{R}(t)\left(1+|x|^{2}\right)
$$

for all $t \in[0, T], x \in \mathbb{R}^{d}$ and $|y| \leq R$.

Remark 2.1 Note that conditions (C2) and (C4) imply the existence of a $K_{R} \in \mathbb{L}$ such that

$$
\sup _{|x| \leq R} \sup _{|y| \leq R}|\alpha(t, y, x)|^{2} \leq K_{R}(t)
$$

for all $t \in[0, T]$.

Theorem 2.1 Let us assume that conditions $\left(C_{1}\right)-\left(C_{4}\right)$ hold, then there exists a unique process $\{X(t)\}_{t \in[0, T]}$ that satisfies equation (2.1).

For $n \geq 1$, consider the following Euler scheme for equation (2.1)

$$
d X_{n}(t)=\beta\left(t, Y_{n}(t), X_{n}\left(\kappa_{n}(t)\right)\right) d t+\alpha\left(t, Y_{n}(t), X_{n}\left(\kappa_{n}(t)\right)\right) d W_{t}
$$

for $t \in[0, T]$, where $X_{n}(t)=\xi(t)$ on $[-C, 0], Y_{n}(t):=\left(X_{n}\left(\delta_{1}(t)\right), \ldots, X_{n}\left(\delta_{k}(t)\right)\right)$ and $\kappa_{n}(t):=[n t] / n$. Note that if $\left(C_{2}\right)$ and Remark 2.1 hold, then (2.2) is well-defined. In addition, consider the condition below:

$\left(C_{5}\right)$ The functions $\beta(t, y, x)$ and $\alpha(t, y, x)$ are continuous in $y$ uniformly in $x$ from compacts, i.e. for every $R>0$ and $t \in[0, T]$,

$$
\sup _{|x| \leq R}\left[\left|\beta(t, y, x)-\beta\left(t, y^{\prime}, x\right)\right|+\left|\alpha(t, y, x)-\alpha\left(t, y^{\prime}, x\right)\right|\right] \rightarrow 0 \quad \text { as } y \rightarrow y^{\prime} .
$$


Theorem 2.2 Let us assume that conditions $\left(C_{1}\right)-\left(C_{5}\right)$ hold. Consider equation (2.1) and the corresponding Euler scheme defined by (2.2). Then

$$
\sup _{t \leq T}\left|X_{n}(t)-X(t)\right| \stackrel{\mathbb{P}}{\rightarrow} 0 \quad \text { as } n \rightarrow \infty .
$$

Let $\left\{X_{n}\right\}_{n \geq 1}$ be a sequence of almost surely finite random variables and $\left\{a_{n}\right\}_{n=1}^{\infty}$ be a positive numerical sequence. Then

$$
X_{n}=\mathcal{O}\left(a_{n}\right)
$$

denotes that there exists an almost surely finite random variable $\zeta$ such that, almost surely,

$$
\left|X_{n}\right| \leq \zeta a_{n}
$$

for any $n \geq 1$. In order to obtain an estimate for the a.s convergence of the Euler scheme, we consider the following conditions:

$\left(A_{1}\right)$ For every $R>0$, there exists a constant $k_{R}$ such that, for all $t \in[0, T]$,

$$
\sup _{|x| \leq R|y| \leq R} \sup _{\mid}(|\beta(t, y, x)|+|\alpha(t, y, x)|) \leq k_{R}
$$

$\left(A_{2}\right)$ For every $R>0$, there exists a constant $c_{R}$ such that, for every $t \in[0, T]$,

$$
\begin{gathered}
\left|\beta(t, y, x)-\beta\left(t, y^{\prime}, x^{\prime}\right)\right| \leq c_{R}\left(\left|y-y^{\prime}\right|+\left|x-x^{\prime}\right|\right) \\
\left|\alpha(t, y, x)-\alpha\left(t, y^{\prime}, x^{\prime}\right)\right|^{2} \leq c_{R}\left(\left|y-y^{\prime}\right|^{2}+\left|x-x^{\prime}\right|^{2}\right)
\end{gathered}
$$

whenever $|x|,\left|x^{\prime}\right|,|y|,\left|y^{\prime}\right|<R$.

$\left(A_{3}\right)$ For every $R>0$, there exists a constant $c_{R}$ such that, for every $t \in[0, T]$,

$$
\begin{aligned}
2\left(x-x^{\prime}\right) & \left(\beta(t, y, x)-\beta\left(t, y, x^{\prime}\right)\right) \leq c_{R}\left|x-x^{\prime}\right|^{2} \\
\left|\beta(t, y, x)-\beta\left(t, y^{\prime}, x\right)\right| & \leq c_{R}\left|y-y^{\prime}\right|
\end{aligned}
$$

whenever $|x|,\left|x^{\prime}\right|,|y|,\left|y^{\prime}\right|<R$.

Remark 2.2 Note that conditions (A1) and (A2) imply that equation (2.1) has a unique (complete) local solution. The same is true if (2.3) is replaced by (A3).

Theorem 2.3 Let conditions $\left(A_{1}\right)$ and $\left(A_{2}\right)$ hold. Assume that equation (2.1) admits a solution $\{X(t)\}_{t \in[0, T]}$. Let $\left\{X_{n}(t)\right\}_{t \in[0, T]}$ denote the solution of the Euler scheme (2.2). Then,

$$
\sup _{t \leq T}\left|X(t)-X_{n}(t)\right|=\mathcal{O}\left(n^{-\gamma}\right) \quad \text { (a.s.) }
$$

for every $\gamma<1 / 2$. Moreover, if one replaces (2.3) with (A3), then (2.4) holds for every $\gamma<1 / 4$

Remark 2.3 Note that without loss of generality, it is assumed henceforth that $T$ is a multiple of $\tau$. To see this, one considers equation (2.1) for every $t \leq T^{\prime}$, where $T^{\prime}=N \tau \geq$ $T$ and $N$ is a positive integer, and observes that all the above conditions are satisfied when $\beta$ and $\alpha$ are replaced by $\beta \mathbb{1}_{\{t \leq T\}}$ and $\alpha \mathbb{1}_{\{t \leq T\}}$. 


\section{Existence and Uniqueness}

Let $b(t, x)$ and $\sigma(t, x)$ be $\mathcal{P} \otimes \mathcal{B}\left(\mathbb{R}^{d}\right)$-measurable functions with values in $\mathbb{R}^{d}$ and $\mathbb{R}^{d \times m}$ respectively. Let $t_{0}$ and $t_{1}$ be any positive constants such that $0 \leq t_{0}<t_{1} \leq T$. Let also $\mathcal{A}$ denote the set of nonnegative $\mathbb{F}$-adapted stochastic processes $L=\{L(t)\}_{t \in[0, T]}$ such that

$$
\int_{0}^{T} L(t) d t<\infty \quad(\text { a.s. }) \text {. }
$$

Consider

$$
d X(t)=b(t, X(t)) d t+\sigma(t, X(t)) d W_{t}, \forall t \in\left[t_{0}, t_{1}\right]
$$

with an initial condition $X\left(t_{0}\right)$ which is an $\mathcal{F}_{t_{0}}$-measurable, almost surely finite random variable. Furthermore, consider conditions

$\left(D_{1}\right)$ The function $b(t, x)$ is continuous in $x$ for any $t$ and $\omega$.

$\left(D_{2}\right)$ For every $R>0$, there exists $\mathcal{K}_{R} \in \mathcal{A}$ such that, almost surely,

$$
\sup _{|x| \leq R}|b(t, x)| \leq \mathcal{K}_{R}(t)
$$

for any $t \in\left[t_{0}, t_{1}\right]$.

$\left(D_{3}\right)$ For every $R>0$, there exists $\mathcal{L}_{R} \in \mathcal{A}$ such that, almost surely,

$$
2(x-z)(b(t, x)-b(t, z))+|\sigma(t, x)-\sigma(t, z)|^{2} \leq \mathcal{L}_{R}(t)|x-z|^{2}
$$

for any $t \in\left[t_{0}, t_{1}\right]$ and $|x|,|z|<R$.

$\left(D_{4}\right)$ There exists $\mathcal{M} \in \mathcal{A}$ such that, almost surely,

$$
2 x b(t, x)+|\sigma(t, x)|^{2} \leq \mathcal{M}(t)\left(1+|x|^{2}\right)
$$

for every $t \in\left[t_{0}, t_{1}\right]$ and $x \in \mathbb{R}^{d}$.

The following existence and uniqueness theorem is known from [5] and [11].

Theorem 3.1 Let us assume that conditions $\left(D_{1}\right)-\left(D_{4}\right)$ hold, then there exists a unique process $\{X(t)\}_{t \in\left[t_{0}, t_{1}\right]}$ that satisfies equation (3.1).

We are ready now to proceed with the proof of the main theorem of this section.

Proof of Theorem 2.1. One considers first the interval $[0, \tau)$ and observes that this reduces to the well-known case of stochastic differential equations (without delay) where the assumptions $\left(C_{1}\right)-\left(C_{4}\right)$ guarantee the existence of a unique, continuous solution (see Theorem 3.1 above). One then observes that

$$
X(\tau):=X(0)+\int_{0}^{\tau} \beta(t, Y(t), X(t)) d t+\int_{0}^{\tau} \alpha(t, Y(t), X(t)) d W_{t}
$$

with $Y(t):=\left(X\left(\delta_{1}(t)\right), \ldots, X\left(\delta_{k}(t)\right)\right)$, is well defined. Inductively, one may assume that a unique, continuous solution exists on the interval $[(i-1) \tau, i \tau]$, for some positive integer 
$i \in\{1, \ldots, N\}$, with the aim to prove that the same is true on $[i \tau,(i+1) \tau]$. One then considers equation (3.1) with

$$
b(t, x):=\beta(t, Y(t), x), \quad \sigma(t, x):=\alpha(t, Y(t), x)
$$

for every $t \in[i \tau,(i+1) \tau)$, and with initial condition $X(i \tau)$ which is an $\mathcal{F}_{i \tau}$-measurable, almost surely finite random variable. One immediately observes that $b(t, x)$ and $\sigma(t, x)$ are $\mathcal{P} \otimes \mathcal{B}\left(\mathbb{R}^{d}\right)$-measurable functions with values in $\mathbb{R}^{d}$ and $\mathbb{R}^{d \times m}$ respectively as a direct consequence of the measurability properties of the aforementioned functions $\beta$ and $\alpha$. Furthermore, one obtains that $(D 1)-(D 4)$ hold for every $t \in[i \tau,(i+1) \tau)$ due to assumptions $(C 1)$-(C4). More precisely, $\left(D_{1}\right)$ is a direct consequence of $\left(C_{1}\right) ;\left(D_{2}\right)$ is a consequence of $\left(C_{2}\right)$ since $\sup _{i \tau \leq t<(i+1) \tau} X\left(\delta_{j}(t)\right)$ is almost surely finite (for $1 \leq j \leq k$ ) and $\mathcal{K}_{R}(t)$ can be given as

$$
\mathcal{K}_{R}(t):=K_{R}(t) \mathbb{I}_{\Omega_{R}}+\sum_{l=R+1}^{\infty} K_{l}(t) \mathbb{I}_{\Omega_{l}^{\prime}},
$$

where

$$
\Omega_{l}:=\left\{\sup _{i \tau \leq t<(i+1) \tau}|Y(t)| \leq l\right\} \quad \text { and } \quad \Omega_{l}^{\prime}:=\Omega_{l+1} \backslash \Omega_{l}
$$

for integers $l \geq 1$. Similarly, one proves that $\left(D_{3}\right)$ is a consequence of $\left(C_{3}\right)$. Clearly, $\left(D_{4}\right)$ holds with

$$
\mathcal{M}:=\sum_{l=1}^{\infty} M_{l} \mathbb{1}_{\Omega_{l}^{\prime}} \in \mathcal{A}
$$

where $M_{l}$ is from $\left(C_{4}\right)$. Finally, Theorem 3.1 is used here so as to obtain a unique solution on $[i \tau,(i+1) \tau)$. Then, one observes that

$$
X((i+1) \tau):=X(i \tau)+\int_{i \tau}^{(i+1) \tau} \beta(t, Y(t), X(t)) d t+\int_{i \tau}^{(i+1) \tau} \alpha(t, Y(t), X(t)) d W_{t}
$$

is well-defined, and that concludes the induction, and consequently, the proof is complete.

\section{Convergence in Probability}

For each integer $n \geq 1$, let $b_{n}=b_{n}(t, x)$ and $\sigma_{n}=\sigma_{n}(t, x)$ be $\mathcal{P} \otimes \mathcal{B}\left(\mathbb{R}^{d}\right)$-measurable functions with values in $\mathbb{R}^{d}$ and $\mathbb{R}^{d \times m}$ respectively. Let $t_{0}$ and $t_{1}$ be positive constants such that $0 \leq t_{0}<t_{1} \leq T$. Moreover, consider the following Euler scheme

$$
d X_{n}(t)=b_{n}\left(t, X_{n}\left(\kappa_{n}(t)\right)\right) d t+\sigma_{n}\left(t, X_{n}\left(\kappa_{n}(t)\right)\right) d W_{t}, \quad \forall t \in\left[t_{0}, t_{1}\right],
$$

where $X_{n}\left(t_{0}\right)=X_{n 0}$ is an $\mathcal{F}_{t_{0}}$-measurable random variable and $\kappa_{n}(t):=[n t] / n$.

In order to prove Theorem 2.2. first we present a slight generalisation of a result from [1] on Euler approximations of stochastic differential equations. 
Theorem 4.1 Consider the Euler scheme (4.1) for equation (3.1). Let conditions ( $\left.D_{1}\right)$ $\left(D_{4}\right)$ hold. Moreover, assume that for every $R>0$ there exists $L_{n R} \in \mathcal{A}$ such that

$$
\sup _{|x| \leq R}\left[\left|b_{n}(t, x)-b(t, x)\right|+\left|\sigma_{n}(t, x)-\sigma(t, x)\right|^{2}\right] \leq L_{n R}(t) \quad \text { (a.s.) }
$$

and

$$
\int_{0}^{T} L_{n R}(t) d t \stackrel{\mathbb{P}}{\rightarrow} 0 \quad \text { as } n \rightarrow \infty .
$$

Finally, let $X_{n 0} \stackrel{\mathbb{P}}{\rightarrow} X\left(t_{0}\right)$ as $n \rightarrow \infty$. Then

$$
\sup _{t_{0} \leq t \leq t_{1}}\left|X_{n}(t)-X(t)\right| \stackrel{\mathbb{P}}{\rightarrow} 0 \quad \text { as } n \rightarrow \infty .
$$

Proof. One observes first that conditions $\left(D_{2}\right)$ and $\left(D_{4}\right)$ together with (4.2) imply that for every $R>0$, there exists $N_{R}(t) \in \mathcal{A}$ such that

$$
\sup _{|x| \leq R}\left[\left|b_{n}(t, x)\right|+\left|\sigma_{n}(t, x)\right|^{2}\right] \leq N_{R}(t) \quad \text { (a.s.) }
$$

for every $t \in\left[t_{0}, t_{1}\right]$. By introducing

$$
H_{n}(t):=X_{n 0}+\int_{t_{0}}^{t} b\left(u, X_{n}\left(\kappa_{n}(u)\right)\right) d u+\int_{t_{0}}^{t} \sigma\left(u, X_{n}\left(\kappa_{n}(u)\right)\right) d W_{u}
$$

and $p_{n}(t):=X_{n}\left(\kappa_{n}(t)\right)-H_{n}(t)$, one obtains that

$$
H_{n}(t)=H_{n}\left(t_{0}\right)+\int_{t_{0}}^{t} b\left(u, H_{n}(u)+p_{n}(u)\right) d u+\int_{t_{0}}^{t} \sigma\left(u, H_{n}(u)+p_{n}(u)\right) d W_{u}
$$

with $H_{n}\left(t_{0}\right)=X_{n 0}$. Furthermore, by introducing $e_{n}(t):=X_{n}(t)-H_{n}(t)$ and the following stopping time

$$
\tau_{n}(R):=\inf \left\{t \geq t_{0}:\left|H_{n}(t)\right|+\left|e_{n}(t)\right| \geq R / 2\right\}
$$

for any $R>0$, one observes that

$$
\left|X_{n}(t)\right| \leq R / 2 \quad \text { and } \quad\left|p_{n}(t)\right|=\left|X_{n}\left(\kappa_{n}(t)\right)-H_{n}(t)\right| \leq R \quad \text { on }\left(t_{0}, \tau_{n}(R)\right] .
$$

In addition, one calculates that as $n \rightarrow \infty$,

$$
\mathbb{P}\left(\tau_{n}(R) \leq t_{1}, \sup _{t_{0} \leq t \leq \tau_{n}(R)}\left|H_{n}(t)\right| \leq \frac{R}{4}\right) \leq \mathbb{P}\left(\sup _{t_{0} \leq t \leq \tau_{n}(R) \wedge t_{1}}\left|e_{n}(t)\right| \geq \frac{R}{4}\right) \rightarrow 0,
$$

due to (4.2) and known results on convergence of stochastic integrals. Moreover, one observes that $p_{n}(t)=X_{n}\left(\kappa_{n}(t)\right)-X_{n}(t)+e_{n}(t)$, and thus

$$
\mathbb{E} \int_{t_{0}}^{t_{1} \wedge \tau_{n}(R)}\left|p_{n}(t)\right| d t \leq \mathbb{E} \int_{t_{0}}^{t_{1} \wedge \tau_{n}(R)}\left|X_{n}\left(\kappa_{n}(t)\right)-X_{n}(t)\right| d t+\mathbb{E} \int_{t_{0}}^{t_{1} \wedge \tau_{n}(R)}\left|e_{n}(t)\right| d t .
$$


By taking into account property (4.3) and Lebesgue's dominated convergence theorem, one concludes that

$$
\begin{aligned}
\left|X_{n}\left(\kappa_{n}(t)\right)-X_{n}(t)\right| \mathbb{I}_{\left[t_{0}, \tau_{n}(R) \wedge t_{1}\right]} \leq & \mathbb{I}_{\left[t_{0}, \tau_{n}(R) \wedge t_{1}\right]}\left|\int_{\kappa_{n}(t)}^{t} b_{n}\left(u, X_{n}\left(\kappa_{n}(u)\right)\right) d u\right| \\
& +\mathbb{I}_{\left[t_{0}, \tau_{n}(R) \wedge t_{1}\right]}\left|\int_{\kappa_{n}(t)}^{t} \sigma_{n}\left(u, X_{n}\left(\kappa_{n}(u)\right)\right) d W_{u}\right|
\end{aligned}
$$

converges to 0 in probability for each $t$, since one observes that

$$
\begin{aligned}
\mathbb{1}_{\left[t_{0}, \tau_{n}(R) \wedge t_{1}\right]} \int_{\kappa_{n}(t)}^{t}\left|\sigma_{n}\left(u, X_{n}\left(\kappa_{n}(u)\right)\right)\right|^{2} d u & \leq \int_{\kappa_{n}(t) \wedge \tau_{n}(R) \wedge t_{0}}^{t \wedge \tau_{n}(R) \wedge t_{1}}\left|\sigma_{n}\left(u, X_{n}\left(\kappa_{n}(u)\right)\right)\right|^{2} d u \\
& =\int_{t_{0}}^{t_{1}} \mathbb{I}_{A_{n}}\left|\sigma_{n}\left(u, X_{n}\left(\kappa_{n}(u)\right)\right)\right|^{2} d u
\end{aligned}
$$

converges almost surely to 0 as $n \rightarrow \infty$, where $A_{n}:=\left(\kappa_{n}(t) \wedge \tau_{n}(R), t \wedge \tau_{n}(R)\right]$. Hence

$$
\lim _{n \rightarrow \infty} \mathbb{E} \int_{t_{0}}^{t_{1} \wedge \tau_{n}(R)}\left|X_{n}\left(\kappa_{n}(t)\right)-X_{n}(t)\right| d t=\lim _{n \rightarrow \infty} \int_{t_{0}}^{t_{1}} \mathbb{E} \mathbb{I}_{\left(t_{0}, \tau_{n}(R)\right]}\left|X_{n}\left(\kappa_{n}(t)\right)-X_{n}(t)\right| d t=0,
$$

by Lebesgue's dominated convergence theorem, since

$$
\left|X_{n}\left(\kappa_{n}(t)\right)-X_{n}(t)\right| \leq R \quad \text { on }\left(t_{0}, \tau_{n}(R)\right]
$$

Due to equation (4.2) and the application of the dominated convergence theorem

$$
\lim _{n \rightarrow \infty} \mathbb{E} \int_{t_{0}}^{t_{1} \wedge \tau_{n}(R)}\left|e_{n}(t)\right| d t=0
$$

which results in

$$
\lim _{n \rightarrow \infty} \mathbb{E} \int_{t_{0}}^{t_{1} \wedge \tau_{n}(R)}\left|p_{n}(t)\right| d t=0 .
$$

Thus, the corresponding conditions of Lemma 2 in Krylov [11] are satisfied and, therefore,

$$
\sup _{t_{0} \leq t \leq t_{1}}\left|H_{n}(t)-H(t)\right| \stackrel{\mathbb{P}}{\rightarrow} 0, \quad \text { as } n \rightarrow \infty
$$

for some process $\{H(t)\}_{t \in\left[t_{0}, t_{1}\right]}$. Furthermore, one calculates that for any $\epsilon>0$,

$$
\mathbb{P}\left(\sup _{t_{0} \leq t \leq t_{1}}\left|e_{n}(t)\right| \geq \epsilon\right) \leq \mathbb{P}\left(\sup _{t_{0} \leq t \leq \tau_{n}(R) \wedge t_{1}}\left|e_{n}(t)\right| \geq \epsilon\right)+\mathbb{P}\left(\tau_{n}(R) \leq t_{1}\right)
$$

Moreover,

$$
\begin{aligned}
\mathbb{P}\left(\tau_{n}(R) \leq t_{1}\right) & \leq \mathbb{P}\left(\sup _{t_{0} \leq t \leq \tau_{n}(R) \wedge t_{1}}\left\{\left|H_{n}(t)\right|+\left|e_{n}(t)\right|\right\} \geq \frac{R}{2}\right) \\
& \leq \mathbb{P}\left(\sup _{t_{0} \leq t \leq t_{1}}\left|H_{n}(t)\right| \geq \frac{R}{4}\right)+\mathbb{P}\left(\sup _{t_{0} \leq t \leq \tau_{n}(R) \wedge t_{1}}\left|e_{n}(t)\right| \geq \frac{R}{4}\right),
\end{aligned}
$$


which implies, by taking into account (4.5)

$$
\limsup _{n \rightarrow \infty} \mathbb{P}\left(\tau_{n}(R) \leq t_{1}\right) \leq \limsup _{n \rightarrow \infty} \mathbb{P}\left(\sup _{t_{0} \leq t \leq t_{1}}\left|H_{n}(t)\right| \geq \frac{R}{4}\right)=\mathbb{P}\left(\sup _{t_{0} \leq t \leq t_{1}}|H(t)| \geq \frac{R}{4}\right)
$$

for all $R>0$ apart from countably many. Letting $R=R_{k} \rightarrow \infty$, for points $R_{k}$ where (4.9) holds, one obtains

$$
\lim _{R_{k} \rightarrow \infty} \limsup _{n \rightarrow \infty} \mathbb{P}\left(\tau_{n}(R) \leq t_{1}\right)=0 .
$$

Thus, by letting $n \rightarrow \infty$ and then $R \uparrow \infty$ in (4.8), one further obtains that

$$
\lim _{n \rightarrow \infty} \mathbb{P}\left(\sup _{t_{0} \leq t \leq t_{1}}\left|e_{n}(t)\right| \geq \epsilon\right)=0 .
$$

As a result,

$$
\sup _{t_{0} \leq t \leq t_{1}}\left|X_{n}(t)-H(t)\right| \leq \sup _{t_{0} \leq t \leq t_{1}}\left|e_{n}(t)\right|+\sup _{t_{0} \leq t \leq t_{1}}\left|H_{n}(t)-H(t)\right| \stackrel{\mathbb{P}}{\rightarrow} 0, \quad \text { as } n \rightarrow \infty .
$$

Furthermore

$$
\int_{t_{0}}^{t_{1}}\left|b_{n}\left(u, X_{n}\left(\kappa_{n}(u)\right)\right)-b(u, H(u))\right| d u \stackrel{\mathbb{P}}{\rightarrow} 0, \quad \text { as } n \rightarrow \infty,
$$

since

$$
\int_{t_{0}}^{t_{1}}\left|b_{n}\left(u, X_{n}\left(\kappa_{n}(u)\right)\right)-b\left(u, X_{n}\left(\kappa_{n}(u)\right)\right)\right| d u \stackrel{\mathbb{P}}{\rightarrow} 0, \quad \text { as } n \rightarrow \infty,
$$

due to (4.2), and

$$
\int_{t_{0}}^{t_{1}}\left|b\left(u, X_{n}\left(\kappa_{n}(u)\right)\right)-b(u, H(u))\right| d u \stackrel{\mathbb{P}}{\rightarrow} 0, \quad \text { as } n \rightarrow \infty,
$$

due to the continuity of $b(t, x)$ in $x,(4.10),\left(D_{2}\right)$ and the application of Lebesgue's dominated convergence theorem. More precisely, equation (4.11) holds since

$$
\begin{aligned}
\mathbb{P}\left(\int_{t_{0}}^{t_{1}} \mid b_{n}\left(u, X_{n}\left(\kappa_{n}(u)\right)\right)\right. & -b\left(u, X_{n}\left(\kappa_{n}(u)\right) \mid d u>\epsilon\right) \leq \mathbb{P}\left(\rho_{n}(R) \leq t_{1}\right)+ \\
& \mathbb{P}\left(\int_{t_{0}}^{t_{1} \wedge \rho_{n}(R)} \mid b_{n}\left(u, X_{n}\left(\kappa_{n}(u)\right)\right)-b\left(u, X_{n}\left(\kappa_{n}(u)\right) \mid d u>\epsilon\right)\right.
\end{aligned}
$$

for any $\epsilon>0$ and $R>0$, where

$$
\rho_{n}(R):=\inf \left\{t \geq t_{0}:\left|X_{n}(t)\right| \geq R\right\} .
$$

One then observes that due to (4.2),

$$
\begin{aligned}
\mathbb{P}\left(\int_{t_{0}}^{t_{1} \wedge \rho_{n}(R)} \mid b_{n}\left(u, X_{n}\left(\kappa_{n}(u)\right)\right)-b\left(u, X_{n}\left(\kappa_{n}(u)\right) \mid d u>\epsilon\right) \leq\right. \\
\mathbb{P}\left(\int_{t_{0}}^{t_{1}} \sup _{|x| \leq R}\left|b_{n}(u, x)-b(u, x)\right| d u>\epsilon\right) \rightarrow 0, \quad \text { as } n \rightarrow \infty .
\end{aligned}
$$


Moreover,

$$
\begin{aligned}
\mathbb{P}\left(\rho_{n}(R) \leq t_{1}\right) & \leq \mathbb{P}\left(\sup _{t_{0} \leq t \leq t_{1}}\left|X_{n}(t)\right| \geq R\right) \\
& \leq \mathbb{P}\left(\sup _{t_{0} \leq t \leq t_{1}}\left|X_{n}(t)-H(t)\right| \geq \frac{R}{2}\right)+\mathbb{P}\left(\sup _{t_{0} \leq t \leq t_{1}}|H(t)| \geq \frac{R}{2}\right)
\end{aligned}
$$

which yields, due to (4.10), that

$$
\lim _{R \rightarrow \infty} \limsup _{n \rightarrow \infty} \mathbb{P}\left(\rho_{n}(R) \leq t_{1}\right)=0 .
$$

One similarly proves that

$$
\int_{t_{0}}^{t_{1}}\left|\sigma_{n}\left(u, X_{n}\left(\kappa_{n}(u)\right)\right)-\sigma(u, H(u))\right|^{2} d u \stackrel{\mathbb{P}}{\rightarrow} 0, \quad \text { as } n \rightarrow \infty .
$$

In other words, the Euler scheme converges in probability to $H(t)$, uniformly in $t \in\left[t_{0}, t_{1}\right]$, and $H(t)$ satisfies

$$
d H(t)=b(t, H(t)) d t+\sigma(t, H(t)) d W_{t}, \forall t \in\left[t_{0}, t_{1}\right]
$$

which yields $H(t)=X(t)$ (a.s.) for every $t \in\left[t_{0}, t_{1}\right]$. The proof is complete.

We are ready now to proceed with the proof of the main result of this section.

Proof of Theorem 2.2. We prove the theorem by showing that

$$
\sup _{(i-1) \tau \leq t \leq i \tau}\left|X_{n}(t)-X(t)\right| \stackrel{\mathbb{P}}{\rightarrow} 0 \quad \text { as } n \rightarrow \infty
$$

for every $i \in\{1, \ldots, N\}$. For $i=1$, the problem reduces to the well-known case of stochastic differential equations (without delay) where the assumptions $\left(C_{1}\right)-\left(C_{5}\right)$ are enough to prove the result. Furthermore, assume (4.12) is true for $i<N$. Then, as noted in the proof of Theorem 2.1, conditions $\left(C_{1}\right)-\left(C_{4}\right)$ imply that $\left(D_{1}\right)-\left(D_{4}\right)$ hold for $b$ and $\sigma$ as defined in (3.2) with $t_{0}=i \tau$ and $t_{1}=(i+1) \tau$. Moreover, consider

$$
b_{n}(t, x):=\beta\left(t, Y_{n}(t), x\right), \quad \sigma_{n}(t, x):=\alpha\left(t, Y_{n}(t), x\right) .
$$

where $Y_{n}(t):=\left(X_{n}\left(\delta_{1}(t)\right), \ldots, X_{n}\left(\delta_{k}(t)\right)\right)$. Then, condition $\left(C_{5}\right)$ implies that (4.2) holds since for every $t \in[i \tau,(i+1) \tau)$ and $R>0$,

$$
\sup _{|x| \leq R}\left[\left|\beta\left(t, Y_{n}(t), x\right)-\beta(t, Y(t), x)\right|+\left|\alpha\left(t, Y_{n}(t), x\right)-\alpha(t, Y(t), x)\right|^{2}\right] \stackrel{\mathbb{P}}{\rightarrow} 0 \quad \text { as } n \rightarrow \infty .
$$

Thus, the application of Lebesgue's dominated convergence theorem, due to $\left(C_{2}\right)$ and Remark 2.1, yields that, for every $R>0$,

$\mathbb{P}\left(\int_{i \tau}^{(i+1) \tau} \sup _{|x| \leq R}\left[\left|\beta\left(t, Y_{n}(t), x\right)-\beta(t, Y(t), x)\right|+\left|\alpha\left(t, Y_{n}(t), x\right)-\alpha(t, Y(t), x)\right|^{2}\right] d t>\epsilon\right) \rightarrow 0$,

as $n \rightarrow \infty$. Consequently, in light of Theorem 4.1, one concludes that the inductive step is correct and thus the desired result is obtained. 


\section{Rate of convergence}

Let $\left\{X_{n}\right\}_{n \geq 1}$ be a sequence of almost surely finite random variables and $\left\{b_{n}\right\}_{n=1}^{\infty}$ be a positive numerical sequence. Then

$$
X_{n}=o\left(b_{n}\right)
$$

denotes that there exists a sequence of random variables $\left\{\eta_{n}\right\}_{n \geq 1}$ converging to 0 almost surely, such that

$$
\left|X_{n}\right| \leq \eta_{n} b_{n} \quad \text { (a.s.) for any } n \geq 1 \text {. }
$$

A useful lemma follows that originates from the Gyöngy and Krylov [5].

Lemma 5.1 Let $X_{n}:=\left\{X_{n}(t)\right\}_{t \in[0, T]}$ be a cadlag stochastic process taking values in $\mathbb{R}^{k}$ for every integer $n \geq 1$. Define

$$
\tau_{n \epsilon}=\inf \left\{t \in[0, T]:\left|X_{n}(t)\right| \geq \epsilon\right\}, \quad X_{n \epsilon}(t)=X_{n}\left(t \wedge \tau_{n \epsilon}\right)
$$

for some $\epsilon>0$. Then, the following statements hold:

(i) If $\sup _{t \in[0, T]}\left|X_{n \epsilon}(t)\right| \rightarrow 0$ in probability, then $\sup _{t \in[0, T]}\left|X_{n}(t)\right| \rightarrow 0$ in probability as well.

(ii) If $\sup _{t \in[0, T]}\left|X_{n \epsilon}(t)\right| \rightarrow 0$ almost surely, then $\sup _{t \in[0, T]}\left|X_{n}(t)\right| \rightarrow 0$ almost surely as well.

(iii) If $\sup _{t \in[0, T]}\left|X_{n \epsilon}(t)\right|=\mathcal{O}\left(a_{n}\right)$ for a numerical sequence $0<a_{n} \rightarrow 0$, then $\sup _{t \in[0, T]}\left|X_{n}(t)\right|=$ $\mathcal{O}\left(a_{n}\right)$ as well.

Proof. See Lemma 3.5 in Gyöngy and Shmatkov [7].

Lemma 5.2 Let $T \in[0, \infty)$ and let $f:=\left\{f_{t}\right\}_{t \in[0, T]}$ and $g:=\left\{g_{t}\right\}_{t \in[0, T]}$ be non-negative continuous $\mathbb{F}$-adapted processes such that, for any constant $c>0$,

$$
\mathbb{E}\left[f_{\tau} \mathbb{1}_{\left\{g_{0} \leq c\right\}}\right] \leq \mathbb{E}\left[g_{\tau} \mathbb{1}_{\left\{g_{0} \leq c\right\}}\right]
$$

for any stopping time $\tau \leq T$. Then, for any stopping time $\tau \leq T$ and $\gamma \in(0,1)$,

$$
\mathbb{E}\left[\sup _{t \leq \tau} f_{t}^{\gamma}\right] \leq \frac{2-\gamma}{1-\gamma} \mathbb{E}\left[\sup _{t \leq \tau} g_{t}^{\gamma}\right]
$$

Proof. See [12] and also Gyöngy and Krylov [6].

The proof of the following lemma is an easy exercise left for the reader.

Lemma 5.3 Let $X_{n}=\left\{X_{n}(t)\right\}_{t \in[0, T]}$ be a cadlag stochastic process taking values in $\mathbb{R}^{k}$ for every integer $n \geq 1$, and let $\left\{a_{n}\right\}_{n=1}^{\infty}$ be a positive numerical sequence. Assume there exists a sequence of stopping times $\left\{\tau_{R}\right\}_{R=1}^{\infty}$, such that $\lim _{R \rightarrow \infty} P\left(\tau_{R}<T\right)=0$, and for each $R$

$$
\sup _{t \leq T}\left|X_{n}\left(t \wedge \tau_{R}\right)\right|=\mathcal{O}\left(a_{n}\right)
$$

Then

$$
\sup _{t \leq T}\left|X_{n}(t)\right|=\mathcal{O}\left(a_{n}\right)
$$


To formulate our next lemma we consider for each integer $n \geq 1$ an Itô process $Z_{n}=$ $\left\{Z_{n}(t)\right\}_{t \in[0, T]}$ with stochastic differential

$$
d Z_{n}(t)=f_{n}(t) d t+g_{n}(t) d W_{t}, \quad t \in[0, T]
$$

where $f_{n}$ and $g_{n}$ are adapted stochastic processes with values in $\mathbb{R}^{d}$ and $\mathbb{R}^{d \times m}$ respectively, such that almost surely

$$
\int_{0}^{T}\left|f_{n}(t)\right| d t<\infty, \quad \int_{0}^{T}\left|g_{n}(t)\right|^{2} d t<\infty .
$$

Lemma 5.4 Let $\gamma>0$ be a fixed number and assume that

$$
Z_{n}(0)=\mathcal{O}\left(n^{-\kappa}\right) \quad \text { for all } \kappa<\gamma
$$

and almost surely

$$
\max \left(Z_{n}(t) f_{n}(t),\left|g_{n}(t)\right|^{2}\right) \leq L_{n}(t)\left|Z_{n}(t)\right|^{2}+\eta_{n}(t), \quad \text { for all } t \in[0, T],
$$

where $L_{n}$ and $\eta_{n}$ are non-negative adapted processes such that

$$
\int_{0}^{T} L_{n}(t) d t=o(\ln n)
$$

and

$$
\int_{0}^{T} \eta_{n}(t) d t=\mathcal{O}\left(n^{-2 \kappa}\right) \quad \text { for any } \kappa<\gamma
$$

Then,

$$
\sup _{t \leq T}\left|Z_{n}(t)\right|=\mathcal{O}\left(n^{-\kappa}\right) \quad \text { for any } \kappa<\gamma
$$

Proof. Let $\kappa \in(0, \gamma)$ and set $\Omega_{R}=\left\{\sup _{n \geq 1}\left|Z_{n}(0)\right| n^{\kappa} \leq R\right\}$. Note that $\Omega_{R}$ is $\mathcal{F}_{0}$ measurable. Then $\lim _{R \rightarrow \infty} P\left(\Omega_{R}\right)=1$ by condition (5.1), i.e., it is enough to prove (5.5) for almost every $\omega \in \Omega_{R}$ for each $R$. Thus by replacing $Z_{n}, f_{n}$ and $g_{n}$ with $R^{-1} \mathbb{1}_{\Omega_{R}} Z_{n}$, $R^{-1} \mathbb{1}_{\Omega_{R}} f_{n}$ and $R^{-1} \mathbb{1}_{\Omega_{R}} g_{n}$, respectively, we see that without loss of generality we may assume that almost surely

$$
\left|Z_{n}(0)\right| \leq n^{-\kappa} \quad \text { for all } n \geq 1
$$

Using this assumption we consider the stopping time $\tau_{n}:=\inf \left\{t \geq 0:\left|Z_{n}(t)\right| \geq 1\right\}$ to obtain that

$$
\sup _{t \leq T}\left|Z_{n}\left(t \wedge \tau_{n}\right)\right| \leq 1, \quad \text { for all } n \geq 1
$$

Thus, by Lemma 5.1, replacing $Z_{n}, f_{n}, g_{n}, L_{n}$ and $\eta_{n}$ with $Z_{n}\left(\cdot \wedge \tau_{n}\right), f_{n} \mathbb{1}_{\left[0, \tau_{n}\right]}, g_{n} \mathbb{1}_{\left[0, \tau_{n}\right]}$, $L_{n} \mathbb{I}_{\left[0, \tau_{n}\right]}$ and $\eta_{n} \mathbb{1}_{\left[0, \tau_{n}\right]}$ respectively, without loss of generality we may assume

$$
\sup _{t \leq T}\left|Z_{n}(t)\right| \leq 1, \quad \text { for all } n \geq 1
$$


Consider for every integer $R \geq 1$ the stopping time

$$
\sigma_{R}:=\inf \left\{t \geq 0: \sup _{n \geq 1} n^{2 \kappa} \int_{0}^{t} \eta_{n}(s) d s \geq R^{2}\right\} \wedge T
$$

to get

$$
\int_{0}^{\sigma_{R}} \eta_{n}(s) d s \leq R^{2} n^{-2 \kappa}, \quad \text { for all } n \geq 1 .
$$

Due to condition (5.4) we have $\lim _{R \rightarrow \infty} P\left(\sigma_{R}<T\right)=0$. Hence by virtue of Lemma 5.3 we need only show (5.5) for $Z_{n}\left(\cdot \wedge \sigma_{R}\right)$, for each $R$, in place of $Z_{n}$. Thus using $R^{-1} Z_{n}\left(\cdot \wedge \sigma_{R}\right)$, $R^{-1} f_{n} \mathbb{I}_{\left[0, \sigma_{R}\right]}, R^{-1} g_{n} \mathbb{I}_{\left[0, \sigma_{R}\right]}, L_{n} \mathbb{I}_{\left[0, \sigma_{R}\right]}$ and $R^{-2} \eta_{n} \mathbb{I}_{\left[0, \sigma_{R}\right]}$ in place of $Z_{n}, f_{n}, g_{n}, L_{n}$ and $\eta_{n}$ respectively, without loss of generality we may assume

$$
\int_{0}^{T} \eta_{n}(s) d s \leq n^{-2 \kappa}, \quad \text { for all } n \geq 1 .
$$

Introduce finally the stopping times

$$
\rho_{N}^{\epsilon}:=\inf \left\{t \geq 0: \sup _{n \geq N} \frac{\int_{0}^{t} L_{n}(s) d s}{\ln n} \geq \epsilon\right\} \wedge T,
$$

for integers $N \geq 2$ and for any (small) $\epsilon>0$. Then

$$
\exp \left(\int_{0}^{\rho_{N}^{\epsilon}} L_{n}(s) d s\right) \leq n^{\epsilon}, \quad \text { for all } n \geq N,
$$

which implies that the random variable

$$
\psi_{\epsilon}^{N}:=\sup _{n \geq 1} n^{-\epsilon} \exp \left(\int_{0}^{\rho_{N}^{\epsilon}} L_{n}(s) d s\right)
$$

is almost surely finite and we have

$$
\exp \left(\int_{0}^{\rho_{N}^{\epsilon}} L_{n}(s) d s\right) \leq \psi_{\epsilon}^{N} n^{\epsilon}, \quad \text { for all } n \geq 1 .
$$

Due to condition (5.3) $\lim _{N \rightarrow \infty} P\left(\rho_{N}^{\epsilon}<T\right)=0$. Thus using Lemma 5.3 as before, we can see that without loss of generality we may assume that for any small $\varepsilon>0$ there is a finite random variable $\psi_{\varepsilon}$ such that almost surely

$$
\exp \left(\int_{0}^{T} L_{n}(s) d s\right) \leq \psi_{\epsilon} n^{\epsilon}, \quad \text { for all } n \geq 1
$$

holds. Now we prove the lemma under the additional conditions (5.6) through (5.9). Set

$$
\phi_{n}(t):=\exp \left(-(2 r+1) \int_{0}^{t} L_{n}(s) d s\right) .
$$


Then, for any $r \geq 2$, Ito's formula yields

$$
\begin{aligned}
d\left(\phi_{n}(t)\left|Z_{n}(t)\right|^{2}\right)^{r}= & 2 r \phi_{n}^{r}(t)\left(\left|Z_{n}(t)\right|^{2(r-1)} Z_{n}(t) d Z_{n}(t)+(r-1)\left|Z_{n}(t)\right|^{2(r-2)}\left|g_{n}^{T}(t) Z_{n}(t)\right|^{2} d t\right. \\
& \left.+\frac{1}{2}\left|Z_{n}(t)\right|^{2(r-1)}\left|g_{n}(t)\right|^{2} d t\right)+\left|Z_{n}(t)\right|^{2 r} d \phi_{n}^{r}(t) \\
\leq & r \phi_{n}^{r}(t)\left|Z_{n}(t)\right|^{2(r-1)}\left(2 Z_{n}(t) d Z_{n}(t)+(2 r-1)\left|g_{n}(t)\right|^{2} d t\right) \\
& -r(2 r+1) L_{n}(t) \phi_{n}^{r}(t)\left|Z_{n}(t)\right|^{2 r} d t .
\end{aligned}
$$

Hence by (5.2),

$d\left(\phi_{n}(t)\left|Z_{n}(t)\right|^{2}\right)^{r} \leq r(2 r+1) \phi_{n}^{r}(t)\left|Z_{n}(t)\right|^{2(r-1)} \eta_{n}(t) d t+2 r\left|Z_{n}(t)\right|^{2(r-1)} \phi_{n}^{r}(t) Z_{n}(t) g_{n}(t) d W_{t}$ on $[0, T]$. Thus, for every stopping time $\tau \leq T$

$$
\mathbb{E}\left(\phi_{n}(\tau)\left|Z_{n}(\tau)\right|^{2}\right)^{r} \leq n^{-2 r \kappa}+r(2 r+1) \mathbb{E} \int_{0}^{\tau}\left(\phi_{n}(t)\left|Z_{n}(t)\right|^{2}\right)^{r-1} \eta_{n}(t) d t
$$

Then, one applies Lemma 5.2 with the non-negative processes $f$ and $g$ being represented by $f_{t}:=\left(\phi_{n}(t)\left|Z_{n}(t)\right|^{2}\right)^{r}$ and

$$
g_{t}:=n^{-2 r \delta \kappa}+r(2 r+1) \int_{0}^{t} \phi_{n}^{r}(s)\left|Z_{n}(s)\right|^{2(r-1)} \eta_{n}(s) d s,
$$

for every $t \in[0, T]$, to obtain that, for any $\delta \in(0,1)$,

$$
\mathbb{E}\left[\sup _{t \leq T}\left(\phi_{n}(t)\left|Z_{n}(t)\right|^{2}\right)^{r \delta}\right] \leq C n^{-2 r \delta \kappa}+C \mathbb{E}\left(\int_{0}^{T}\left(\phi_{n}(t)\left|Z_{n}(t)\right|^{2}\right)^{r-1} \eta_{n}(t) d t\right)^{\delta} .
$$

Hence the application of Young's inequality yields

$$
\mathbb{E}\left[\sup _{t \leq T}\left(\phi_{n}(t)\left|Z_{n}(t)\right|^{2}\right)^{r \delta}\right] \leq C n^{-2 r \delta \kappa}+\frac{1}{2} \mathbb{E} \sup _{t \leq T}\left(\phi_{n}(t)\left|Z_{n}(t)\right|^{2}\right)^{r \delta}+C \mathbb{E}\left(\int_{0}^{T} \eta_{n}(t) d t\right)^{r \delta} .
$$

Thus, due to (5.8) we have

$$
\sum_{n} \mathbb{P}\left(\sup _{t \leq T}\left(\phi_{n}(t)\left|Z_{n}(t)\right|^{2}\right)>n^{-2 \kappa^{\prime}}\right) \leq C \sum_{n} n^{2 r \delta\left(\kappa^{\prime}-\kappa\right)}<\infty
$$

for a sufficiently large $r, \delta \in(0,1)$ and any $\kappa^{\prime}<\kappa<\gamma$. Here and above, $\mathrm{C}$ denotes constants that depend on $r$ and $\delta$ but are independent of $n$. Borel-Cantelli lemma then implies that for each $\kappa<\gamma$ there is a finite random variable $\zeta_{\kappa}$ such that almost surely

$$
\sup _{t \leq T}\left(\phi_{n}(t)\left|Z_{n}(t)\right|^{2}\right) \leq \zeta_{\kappa} n^{-2 \kappa}
$$

for all $n \geq 1$. Hence, due to (5.9)

$$
\sup _{t \leq T}\left|Z_{n}(t)\right|^{2} \leq \zeta_{\kappa} n^{-2 \kappa} \exp \left((2 r+1) \int_{0}^{T} L_{n}(t) d t\right) \leq \psi_{\epsilon} \zeta_{\kappa} n^{\epsilon(2 r+1)-2 \kappa} .
$$

By taking $\epsilon$ sufficiently small and $\kappa$ sufficiently close to $\gamma$, the desired result follows immediately. The proof is complete.

One is then ready to proceed with the calculation of the rate of convergence for the Euler scheme (4.1). Key conditions are described below. 
$\left(E_{1}\right)$ For every $R>0$, there exist finite $\mathcal{F}_{t_{0}}$-measurable random variable $C_{R}$ such that, almost surely,

$$
\begin{gathered}
|b(t, x)-b(t, y)| \leq C_{R}|x-y| \\
|\sigma(t, x)-\sigma(t, y)|^{2} \leq C_{R}|x-y|^{2}
\end{gathered}
$$

for every $t \in\left[t_{0}, t_{1}\right]$ and $|x|,|y|<R$.

$\left(E_{2}\right)$ For every $R>0$, there exist finite $\mathcal{F}_{t_{0}}$-measurable random variables $K_{R}$ such that, almost surely,

$$
\left|b_{n}(t, x)\right| \leq K_{R} \quad \text { and } \quad\left|\sigma_{n}(t, x)\right| \leq K_{R}
$$

for every $n \geq 1, t \in\left[t_{0}, t_{1}\right]$ and $|x|<R$.

$\left(E_{3}\right)$ For every $R>0$, there exist adapted processes $M_{R n}$ such that, almost surely,

$$
\begin{aligned}
& \left|b(t, x)-b_{n}(t, x)\right|^{2} \leq M_{R n}(t) \\
& \left|\sigma(t, x)-\sigma_{n}(t, x)\right|^{2} \leq M_{R n}(t)
\end{aligned}
$$

for every $n \geq 1, t \in\left[t_{0}, t_{1}\right]$ and $|x|<R$, and for every $\gamma<1 / 2$,

$$
\int_{t_{0}}^{t_{1}} M_{R n}(t) d t=\mathcal{O}\left(n^{-2 \gamma}\right) .
$$

$\left(E_{4}\right)$ Alternatively to (5.10), there exists a finite $\mathcal{F}_{t_{0}}$-measurable random variable $C_{R}$ such that, almost surely,

$$
2(x-y)(b(t, x)-b(t, y)) \leq C_{R}|x-y|^{2}
$$

for every $t \in\left[t_{0}, t_{1}\right]$ and $|x|,|y|<R$.

Remark 5.1 Conditions $\left(E_{2}\right)$ and $\left(E_{3}\right)$ imply that for every $R>0$, there exists a process $M_{R} \in \mathcal{A}$ such that, almost surely,

$$
|b(t, x)|^{2} \leq M_{R}(t) \quad \text { and } \quad|\sigma(t, x)|^{2} \leq M_{R}(t) .
$$

for any $t \in\left[t_{0}, t_{1}\right]$ and $|x|<R$. Hence, due to conditions $\left(E_{1}\right)-\left(E_{3}\right)$, equation (3.1) has a unique (complete) local solution. The same is true if (5.10) is replaced by (E $\left.E_{4}\right)$. The existence of a unique (global) solution can be guaranteed by appropriate assumptions on the growth of $b$ and $\sigma$, e.g. by $\left(D_{4}\right)$.

Theorem 5.5 Let conditions $\left(E_{1}\right)-\left(E_{3}\right)$ hold. Assume that equation (3.1) with initial data $X\left(t_{0}\right)$ admits a solution $\{X(t)\}_{t \in\left[t_{0}, t_{1}\right]}$. Let $\left\{X_{n}(t)\right\}_{t \in\left[t_{0}, t_{1}\right]}$ denote the solution of the Euler scheme (4.1) with initial data $X_{n 0}$ such that

$$
\left.\left|X\left(t_{0}\right)-X_{n 0}\right|=\mathcal{O}\left(n^{-\gamma}\right) \quad \text { (a.s. }\right)
$$

for every $\gamma<1 / 2$. Then

$$
\sup _{t_{0} \leq t \leq t_{1}}\left|X(t)-X_{n}(t)\right|=\mathcal{O}\left(n^{-\gamma}\right) \quad \text { (a.s.) }
$$

for every $\gamma<1 / 2$. Moreover, if one replaces condition (5.10) with $\left(E_{4}\right)$, then (5.12) holds for every $\gamma<1 / 4$. In this case, it is sufficient to require that $\left(E_{3}\right)$ and (5.11) hold for every $\gamma<1 / 4$. 
Proof. Due to Lemma 5.1 and 5.3, it suffices to prove that

$$
\sup _{t_{0} \leq t \leq t_{1}}\left|Z_{n}(t)\right|=\mathcal{O}\left(n^{-\gamma}\right) \quad(\text { a.s. })
$$

for every $\gamma<1 / 2$, where

$$
\begin{gathered}
Z_{n}(t):=X\left(t \wedge \tau_{n \epsilon R}\right)-X_{n}\left(t \wedge \tau_{n \epsilon R}\right), \\
\tau_{n \epsilon}:=\inf \left\{t \geq t_{0}:\left|X(t)-X_{n}(t)\right| \geq \epsilon\right\}, \quad \tau_{R}:=\inf \left\{t \geq t_{0}:|X(t)| \geq R-1\right\} .
\end{gathered}
$$

and $\tau_{n \epsilon R}=\tau_{n \epsilon} \wedge \tau_{R}$ for every $R>0$ and arbitrary $\epsilon \in(0,1)$.

Moreover, it is enough to prove our result under the condition that assumption $\left(E_{2}\right)$ holds with a constant $L$ instead of a finite $\mathcal{F}_{t_{0}}$-measurable random variable $K_{R}$. One only needs to replace $b_{n}$ and $\sigma_{n}$ with $b_{n} \mathbb{I}_{\left\{K_{R} \leq L\right\}}$ and $\sigma_{n} \mathbb{I}_{\left\{K_{R} \leq L\right\}}$ respectively, for each (fixed) $R>0$, since

$$
\left[K_{R}<L\right] \in \mathcal{F}_{t_{0}} \quad \text { and } \quad \mathbb{P}\left(\bigcup_{L=1}^{\infty}\left[K_{R}<L\right]\right)=1 .
$$

Then, for $h_{n}(s):=\sigma_{n}\left(s, X_{n}\left(\kappa_{n}(s)\right)\right) \mathbb{1}_{\left\{s \leq \tau_{n \in R}\right\}}$, one has $\left|h_{n}(t)\right| \leq L$ on $\left[t_{0}, t_{1}\right]$ and for $r \geq 2$,

$$
\begin{aligned}
\mathbb{E}\left(\int_{t_{0}}^{t_{1}}\left|\int_{\kappa_{n}(t)}^{t} h_{n}(s) d W_{s}\right| d t\right)^{r} & \leq K \mathbb{E}\left(\int_{t_{0}}^{t_{1}}\left|\int_{\kappa_{n}(t)}^{t} h_{n}(s) d W_{s}\right|^{2 r} d t\right)^{\frac{1}{2}} \quad \text { (Hölder) } \\
& \leq K\left(\int_{t_{0}}^{t_{1}} \mathbb{E}\left|\int_{\kappa_{n}(t)}^{t} h_{n}(s) d W_{s}\right|^{2 r} d t\right)^{\frac{1}{2}} \quad \text { (Jensen) } \\
& \leq K n^{-\frac{r}{2}}
\end{aligned}
$$

holds, where $K$ denote positive constants which are independent of $n$. As a result, due to Markov's inequality,

$$
\sum_{n} \mathbb{P}\left(\int_{t_{0}}^{t_{1}}\left|\int_{\kappa_{n}(t)}^{t} h_{n}(s) d W_{s}\right| d t>n^{-\gamma}\right) \leq K \sum_{n} n^{\gamma r-\frac{r}{2}}<\infty
$$

for a sufficiently large $r$ and $\gamma \in[0,1 / 2)$, which proves that

$$
\int_{t_{0}}^{t_{1}}\left|\int_{\kappa_{n}(t)}^{t} h_{n}(s) d W_{s}\right| d t=\mathcal{O}\left(n^{-\gamma}\right)
$$

by the application of the Borel-Cantelli lemma. Consequently,

$$
\begin{aligned}
\int_{t_{0}}^{t_{1}} \mid X_{n}(t)-X_{n}\left(\left(\kappa_{n}(t)\right) \mid \mathbb{I}_{\left\{t \leq \tau_{n \in R}\right\}} d t\right. & \leq \int_{t_{0}}^{t_{1}}\left|\int_{\kappa_{n}(t)}^{t} \mathbb{I}_{\left\{s \leq \tau_{n \in R}\right\}} b_{n}\left(s, X_{n}\left(\kappa_{n}(s)\right)\right) d s\right| d t \\
& +\int_{t_{0}}^{t_{1}}\left|\int_{\kappa_{n}(t)}^{t} h_{n}(s) d W_{s}\right| d t \\
& =\mathcal{O}\left(n^{-\gamma}\right)
\end{aligned}
$$

for any $\gamma \in[0,1 / 2)$. Furthermore, in order to apply Lemma 5.4, one defines

$$
f_{n}(t):=\mathbb{1}_{T_{n}}\left[b(t, X(t))-b_{n}\left(t, X_{n}\left(\kappa_{n}(t)\right)\right)\right]
$$


and

$$
g_{n}(t):=\mathbb{1}_{T_{n}}\left[\sigma(t, X(t))-\sigma_{n}\left(t, X_{n}\left(\kappa_{n}(t)\right)\right)\right] .
$$

where $T_{n}:=\left(t_{0}, \tau_{n \in R}\right]$. Moreover, one calculates

$$
\begin{aligned}
Z_{n}(t) f_{n}(t) \leq & \left|Z_{n}(t)\right|\left(\left|b(t, X(t))-b\left(t, X_{n}(t)\right)\right|+\left|b\left(t, X_{n}(t)\right)-b\left(t, X_{n}\left(\kappa_{n}(t)\right)\right)\right|\right. \\
& \left.+\left|b\left(t, X_{n}\left(\kappa_{n}(t)\right)\right)-b_{n}\left(t, X_{n}\left(\kappa_{n}(t)\right)\right)\right|\right) \mathbb{I}_{T_{n}} \\
\leq & \left(C_{R}+1\right)\left|Z_{n}(t)\right|^{2}+C_{R}\left|X_{n}(t)-X_{n}\left(\kappa_{n}(t)\right)\right|^{2} \mathbb{I}_{T_{n}} \\
& +\left|b\left(t, X_{n}\left(\kappa_{n}(t)\right)\right)-b_{n}\left(t, X_{n}\left(\kappa_{n}(t)\right)\right)\right|^{2} \mathbb{I}_{T_{n}},
\end{aligned}
$$

and,

$$
\begin{aligned}
\left|g_{n}(t)\right|^{2} \leq & 3\left(\left|\sigma(t, X(t))-\sigma\left(t, X_{n}(t)\right)\right|^{2}+\left|\sigma\left(t, X_{n}(t)\right)-\sigma\left(t, X_{n}\left(\kappa_{n}(t)\right)\right)\right|^{2}\right. \\
& +\mid \sigma\left(t, X_{n}\left(\kappa_{n}(t)\right)-\left.\sigma_{n}\left(t, X_{n}\left(\kappa_{n}(t)\right)\right)\right|^{2}\right) \mathbb{I}_{T_{n}} \\
\leq & C_{R}\left|Z_{n}(t)\right|^{2}+C_{R}\left|X_{n}(t)-X_{n}\left(\kappa_{n}(t)\right)\right|^{2} \mathbb{I}_{T_{n}} \\
& +\mid \sigma\left(t, X_{n}\left(\kappa_{n}(t)\right)-\left.\sigma_{n}\left(t, X_{n}\left(\kappa_{n}(t)\right)\right)\right|^{2} \mathbb{I}_{T_{n}} .\right.
\end{aligned}
$$

which yields

$$
\max \left(Z_{n}(t) f_{n}(t),\left|g_{n}(t)\right|^{2}\right) \leq L_{n}\left|Z_{n}(t)\right|^{2}+\eta_{n}(t), \quad \text { for all } t \in\left[t_{0}, t_{1}\right],
$$

due to (5.13) and $\left(E_{3}\right)$, where $L_{n}:=C_{R}+1$ and

$$
\begin{aligned}
\eta_{n}(t):=\left(C_{R}\left|X_{n}(t)-X_{n}\left(\kappa_{n}(t)\right)\right|^{2}\right. & +\left|b\left(t, X_{n}\left(\kappa_{n}(t)\right)\right)-b_{n}\left(t, X_{n}\left(\kappa_{n}(t)\right)\right)\right|^{2} \\
& +\mid \sigma\left(t, X_{n}\left(\kappa_{n}(t)\right)-\left.\sigma_{n}\left(t, X_{n}\left(\kappa_{n}(t)\right)\right)\right|^{2}\right) \mathbb{I}_{T_{n}}
\end{aligned}
$$

satisfy the conditions of Lemma 5.4 for any $\gamma \in[0,1 / 2)$. Thus, application of Lemma 5.4 yields the desired result (5.12) for any $\gamma \in[0,1 / 2)$. Finally, if assumption (5.10) is replaced by $\left(E_{4}\right)$, then

$$
\begin{aligned}
Z_{n}(t) f_{n}(t)= & \left(X(t)-X_{n}(t)\right)\left[b(t, X(t))-b_{n}\left(t, X_{n}\left(\kappa_{n}(t)\right)\right)\right] \mathbb{I}_{T_{n}} \\
= & \left(X(t)-X_{n}\left(\kappa_{n}(t)\right)\right)\left[b(t, X(t))-b\left(t, X_{n}\left(\kappa_{n}(t)\right)\right)\right] \mathbb{I}_{T_{n}} \\
& +\left(X(t)-X_{n}\left(\kappa_{n}(t)\right)\right)\left[b\left(t, X_{n}\left(\kappa_{n}(t)\right)\right)-b_{n}\left(t, X_{n}\left(\kappa_{n}(t)\right)\right)\right] \mathbb{I}_{T_{n}} \\
& +\left(X_{n}\left(\kappa_{n}(t)\right)-X_{n}(t)\right)\left[b(t, X(t))-b_{n}\left(t, X_{n}\left(\kappa_{n}(t)\right)\right)\right] \mathbb{I}_{T_{n}} \\
\leq & \frac{1}{2} C_{R}\left|X(t)-X_{n}\left(\kappa_{n}(t)\right)\right|^{2} \mathbb{I}_{T_{n}} \\
& +\left|X(t)-X_{n}\left(\kappa_{n}(t)\right)\right|\left|b\left(t, X_{n}\left(\kappa_{n}(t)\right)\right)-b_{n}\left(t, X_{n}\left(\kappa_{n}(t)\right)\right)\right| \mathbb{I}_{T_{n}} \\
& +\left|X_{n}\left(\kappa_{n}(t)\right)-X_{n}(t)\right|\left|b(t, X(t))-b_{n}\left(t, X_{n}\left(\kappa_{n}(t)\right)\right)\right| \mathbb{I}_{T_{n}} \\
\leq & \left(C_{R}+1\right)\left|Z_{n}(t)\right|^{2}+\left(C_{R}+1\right)\left|X_{n}\left(\kappa_{n}(t)\right)-X_{n}(t)\right|^{2} \mathbb{I}_{T_{n}}+\frac{1}{2} M_{R n}(t) \\
& +2\left|X_{n}\left(\kappa_{n}(t)\right)-X_{n}(t)\right| \mathbb{I}_{T_{n}}\left(L+M_{R}^{1 / 2}(t)\right),
\end{aligned}
$$


where $M_{R}$ comes from Remark 5.1. Thus

$$
Z_{n}(t) f_{n}(t) \leq L_{n}\left|Z_{n}(t)\right|^{2}+\eta_{n}(t), \quad \text { for all } t \in\left[t_{0}, t_{1}\right],
$$

where $L_{n}:=C_{R}+1$ and $\eta_{n}$ is a non-negative $\mathbb{F}$-adapted process which satisfy the conditions of Lemma 5.4 for any $\gamma \in[0,1 / 4)$ due to (5.13). Thus the proof is complete.

Remark 5.2 One could further observe that assumptions (5.10) \& $\left(E_{2}\right)$ can be relaxed to allow

$$
|b(t, x)-b(t, y)|^{2} \leq M_{R}(t)|x-y|^{2} \quad \text { and } \quad \sup _{|x| \leq R}\left|b_{n}(t, x)\right|^{2} \leq M_{R}(t)
$$

for some process $M_{R} \in \mathcal{A}$, while Theorem 5.5 remains true.

The proof of the main and final result of this section follows.

Proof of Theorem [2.3. We prove the theorem by showing that

$$
\sup _{(i-1) \tau \leq t \leq i \tau}\left|X(t)-X_{n}(t)\right|=\mathcal{O}\left(n^{-\gamma}\right) \quad(\text { a.s. })
$$

for every $i \in\{1, \ldots, N\}$. For $i=1$, the conditions of Theorem 5.5 are satisfied and thus, estimate (5.16) is achieved on the interval $[0, \tau]$. Assume that estimate (5.16) holds for $i<N$. Then let us show that the conditions of Theorem 5.5 for equations (3.1) and (4.1) hold with initial data $X\left(t_{0}\right)=X(i \tau)$ and $X_{n 0}=X_{n}(i \tau)$ and with $b, \sigma$ and $b_{n}, \sigma_{n}$ given by (3.2) and (4.13) respectively. Clearly, (5.11) is satisfied. Furthermore, assumption $\left(E_{1}\right)$ is satisfied since, for every $R>0$, there exist a constant $c_{R}$ such that

$$
|b(t, x)-b(t, y)| \leq c_{R}|x-y| \quad(\text { a.s. })
$$

and

$$
|\sigma(t, x)-\sigma(t, y)|^{2} \leq c_{R}|x-y|^{2} \quad \text { (a.s.) }
$$

for every $t \in[i \tau,(i+1) \tau)$ and $|x|,|y|<R$, due to $\left(A_{2}\right)$. Moreover, for integers $l \geq 1$, define

$$
\Omega_{l}:=\left\{\sup _{n \geq 1} \sup _{m \tau \leq t<(m+1) \tau}\left|Y_{n}(t)\right| \leq l\right\} \quad \text { and } \quad \Omega_{l}^{\prime}:=\Omega_{l} \backslash \Omega_{l-1}
$$

and observe that $\mathbb{P}\left(\cup_{l} \Omega_{l}^{\prime}\right)=1$ due to (5.16)$)$. Thus assumption $\left(E_{2}\right)$ is satisfied since

$$
\sup _{|x| \leq R}\left|b_{n}(t, x)\right|=\sup _{|x| \leq R}\left|\beta\left(t, Y_{n}(t), x\right)\right| \leq k_{R} \mathbb{I}_{\Omega_{R}}+\sum_{l=R+1}^{\infty} k_{l} \mathbb{I}_{\Omega_{l}^{\prime}}<\infty \text { (a.s.) }
$$

and

$$
\sup _{|x| \leq R}\left|\sigma_{n}(t, x)\right|=\sup _{|x| \leq R}\left|\alpha\left(t, Y_{n}(t), x\right)\right| \leq k_{R} \mathbb{I}_{\Omega_{R}}+\sum_{l=R+1}^{\infty} k_{l} \mathbb{I}_{\Omega_{l}^{\prime}}<\infty \text { (a.s.), }
$$

where $k_{l}$ are constants from $\left(A_{1}\right)$. Finally, observe that

$$
\sup _{|x| \leq R}\left|b(t, x)-b_{n}(t, x)\right|^{2} \leq c_{R}\left|Y(t)-Y_{n}(t)\right|^{2}
$$


and

$$
\sup _{|x| \leq R}\left|\sigma(t, x)-\sigma_{n}(t, x)\right|^{2} \leq c_{R}\left|Y(t)-Y_{n}(t)\right|^{2}
$$

for every $t \in[i \tau,(i+1) \tau)$, due to $\left(A_{2}\right)$. Consequently, $\left(E_{3}\right)$ holds for $\gamma<1 / 2$. The application of Theorem 5.5 shows that the inductive step is correct on $[i \tau,(i+1) \tau]$ which yields the result

$$
\sup _{t \leq T}\left|X(t)-X_{n}(t)\right|=\mathcal{O}\left(n^{-\gamma}\right) \quad \text { (a.s.) }
$$

for every $\gamma<1 / 2$. Moreover, if one replaces (2.3) with $(A 3)$, then (2.4) holds for every $\gamma<1 / 4$ due to the fact that Theorem 5.5 applies for the case where assumption (5.10) is replaced by $\left(E_{4}\right)$.

\section{References}

[1] M. Arriojas, Y. Hu, S.-E Mohammed, G. Pap, A delayed Black and Scholes formula, Stoch. Anal. and Appl., 25 (2007), pp. 471-492.

[2] C. T. H. BAKer, E. BuCKWAR, Numerical analysis of explicit one-step methods for stochastic delay differential equations, LMS J. Comput. Math., 3 (2000), pp. 315-335.

[3] E. Buckwar, R. Kuske, S.-E. Mohammed, T. Shardlow, Weak convergence of the Euler scheme for stochastic differential delay equations, LMS J. Comput. Math., 11 (2008), pp. 60-99.

[4] I. Elsanosi, B. Øksendal, A. Sulem, Some solvable stochastic control problems with delay, Stoch. and Stoch. Rep., 71 (2000), pp. 69-89.

[5] I. GyÖNGy, N.V. KRYLOV, On stochastic equations with respect to semimartingales I, Stoch., 4 (1980), pp. 1-21.

[6] I. Gyöngy, N.V. KRYLOV, On the rate of convergence of splitting-up approximations for SPDEs, Progress Prob., 56 (2003), pp. 301-321.

[7] I. Gyöngy, A. Shmatkov, Rate of convergence of Wong-Zakai approximations for stochastic partial differential equations, Appl. Math. Optim., 54 (2006), pp. 315-341.

[8] D. Higham, X. Mao, C. Yuan, Almost sure and moment exponential stability in the numerical simulation of stochastic differential equations, SIAM J. Numer. Anal., 45 (2007), pp. 592-609.

[9] Y. Hu, S.-E. A. Mohammed, F. YAn, Discrete-time approximations of stochastic delay equations: The Milstein scheme, Ann. Prob., 32 (2004), pp. 265-314.

[10] P.E. Kloeden, A. Neuenkirch, The pathwise convergence of approximation schemes for stochastic differential equations, LMS J. Comput. Math., 10 (2007), pp. 235-253. 
[11] N.V. KRYLOV, A simple proof of the existence of a solution of Itô's equation with monotone coefficients, Theory Probab. Appl., 35 (1990), pp. 583-587.

[12] N.V. Krylov, Introduction to the theory of diffusion processes, Amer. Math. Soc., Providence, RI, 1995.

[13] U. KüChler, E. Platen, Strong discrete time approximation of stochastic differential equations with time delay, Math. Comput. Simulation, 54 (2000), pp. 189-205.

[14] X. Mao, S. Sabanis, Numerical solutions of stochastic differential delay equations under local Lipschitz condition, J. Comput. Appl. Math., 151 (2003), pp. 215-227.

[15] X. MaO, Y. Shen, C. YuAn, Almost surely asymptotic stability of neutral stochastic differential delay equations with Markovian switching, Stoc. Proc. App., 118 (2008), pp. 1385-1406.

[16] X. Mao, C. YuAn, J. Zou, Stochastic differential delay equations of population dynamics, J. Math. Anal. Appl., 304 (2005), pp. 296-320.

[17] N. MCWilliams, S. Sabanis, Arithmetic Asian options under stochastic delay models, Appl. Math. Fin., 18 (2011), pp. 423-446.

[18] M.-K. VOn Renesse, M. Scheutzow, Existence and uniqueness of solutions of stochastic functional differential equations, Random Oper. Stoch. Equ., 18 (2010), pp. 267-284.

[19] F. Wu, X. Mao, L. SzPruch, Almost sure exponential stability of numerical solutions for stochastic delay differential equations, Numer. Math., 115 (2010), pp. 681-697. 